\title{
FRACTURE RESISTANCE AND COLOR STABILITY OF TWO HYBRID CERAMIC VENEERS VERSUS LITHIUM DISILICATE GLASS CERAMIC VENEERS
}

\author{
Marwa Tannir*, Essam Osman ${ }^{* *}$, Mohammad Rayyan ${ }^{* * *}$ and Ehab A. Farghaly****
}

\begin{abstract}
Purpose: To compare the color stability and fracture strength of two hybrid ceramic (Suprinity and Enamic) veneers versus Lithium Disilicate ceramic veneers.
\end{abstract}

Materials and methods: Thirty freshly extracted maxillary central incisors were divided equally into three groups ( $\mathrm{n}=10)$ : group Em (IPS empress II, Ivoclar Vivadent) as a control, group VS (Suprinity, Vita Zahnfabrik), and group VE (Enamic, Vita Zahnfabrik). An incisal reduction of $1.5 \mathrm{~mm}$ was made with a butt-joint margin and $0.6 \mathrm{~mm}$ chamfer finish line was prepared on the labial surface of each specimen and on the middle of the proximal surfaces. Each group received different type of veneers according to the sample grouping.

Using spectrophotometer (Nippon Densmoku industries), $\Delta \mathrm{E}$ was recorded for each specimen before and after 15 days of immersion in a coffee solution considering the value of $\Delta \mathrm{E}=3.3$ as clinically significant staining threshold. All the specimens were subjected to fracture strength test using universal testing machine (The Testometric Company Limited) using cross head speed of 0.5 $\mathrm{mm} / \mathrm{min}$ at 135 degrees' angle. One-way ANOVA and chi-square were used for statistical analysis.

Results: Regarding the mean color stability between studied groups, group VS scored the least $\Delta \mathrm{E}(3.52 \pm 2.46)$ followed by group $\mathrm{VE}(4.14 \pm 1.94)$ then group $\mathrm{Em}(4.49 \pm 2.45)$. On the other hand, group VS scored the highest fracture strength values $(471.04 \pm 274.5)$ followed by group Em $(442.13 \pm 236.1)$ then group VE $(394.72 \pm 160.84)$. One-wayANOVA revealed no significant differences between groups in both color stability $(\mathrm{P}=0.636)$ and fracture strength $(\mathrm{P}=605)$.

Conclusion: The tested hybrid ceramics could present an acceptable treatment option for fabricating ceramic veneers.

\footnotetext{
* Clinical instructor Prosthodontics, Beirut Arab University, Faculty of Dentistry

** Professor of Dental Biomaterials, Beirut Arab University, Faculty of Dentistry

*** Associate Professor of Prosthodontics, Beirut Arab University, Faculty of Dentistry, Assistant Professor of Fixed

Prosthodontics, Faculty of Dentistry, Misr University for Science and Technology, Cairo, Egypt

**** Lecturer of Fixed Prosthodontics, Faculty of Dentistry, Misr University for Science and Technology, Cairo, Egypt
} 


\section{INTRODUCTION}

Dental ceramics comprise a large family of inorganic non- metal materials, and are commonly divided into three groups: i) mostly glassy ceramics (feldspathic porcelain), ii) particle filled ceramics (e.g. e-max) and iii) polycrystalline ceramics (e.g. Zirconia) ${ }^{1}$. Feldspathic veneers are the most esthetic and artistic veneers. A skilled lab technician can build life like teeth shapes and colors using theses materials. Their main draw back is their low flexural strength $(60-70 \mathrm{Mpa})^{2}$, which necessitates excessive removal of tooth structure and possible exposure of dentine islands ${ }^{3}$.

e-max belongs to the particle filled ceramic category and is the most material used and preferred by many clinicians to fabricate veneers. Although it is inferior in esthetics to feldspathic porcelain but a 0.6 mm preparation thickness protocol can be adopted due to its high flexural strength (360-400 Mpa) It can be used as a monolith structure or bilayered but the later may necessitates more removal of valuable bonding enamel structure ${ }^{4}$. Polycrystalline ceramics are superior to all ceramic groups in flexural strength chemical solubility and flexural strength. In the past zirconia was accused of being "white". It had a dull artificial color due to its opaque, glassfree nature. It wasn't indicated for veneers. Its use was limited to coping material in posterior region which will be later veneered by more esthetic ceramics. Recently High translucency zirconia solved the "opaque white" problem and was indicated by manufacturers for esthetic veneers. And due to its high flexural strength it dominated sectors of minimal preparation veneers (Varioneers) ${ }^{5}$. Few years ago, VITA ENAMIC (Vita Zahnfabrik) was introduced. It was the first hybrid dental ceramic with a dual-network structure. Where dominant ceramic network is strengthened by a polymer network ${ }^{6}$. It was said that it combines the best characteristics of a ceramic and a composite. More recently, a new generation of glass ceramic material was present- ed, Vita Suprinity (Vita Zahnfabrik) and Celtra duo (Dentsply). They are the same material but presented by two different companies. In these materials, glass ceramic is enriched with zirconia (approx. $10 \%$ by weight), resulting in zirconia reinforced lithium silicate ceramic (ZLS). This glass ceramic featured a special fine-grained and homogeneous structure, which was claimed by the manufacturer to provide excellent physical qualities, consistent high load capacity, long-term reliability and excellent esthetic properties ${ }^{7}$. Both of the materials were indicated for esthetic veneer restoration as a successful substitute for classical ceramics. The null hypothesis of this study is that there will be no significant difference between the tested groups.

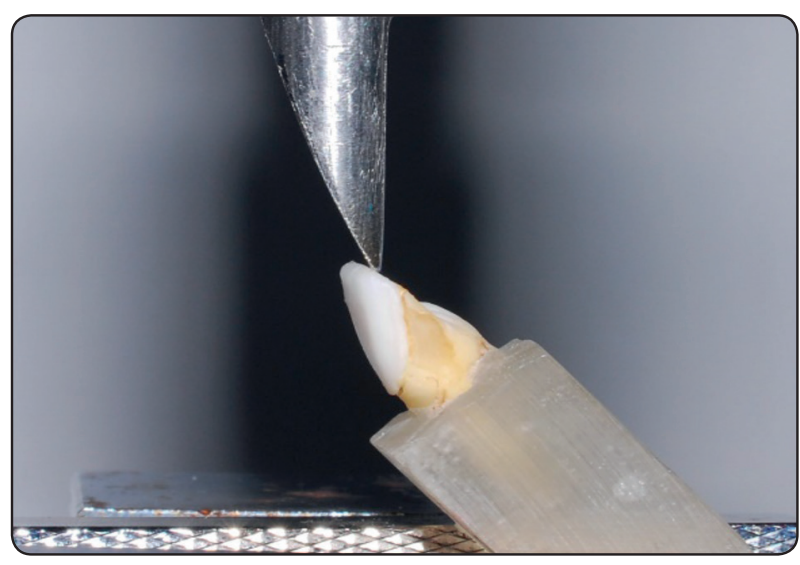

Fig. (1) Mounted specimen under loading.

\section{MATERIALS AND METHODS}

Thirty freshly extracted maxillary central incisors were divided equally into three groups $(n=10)$ : group Em (IPS empress II, Ivoclar Vivadent) as a control, group VS (Suprinity, Vita Zahnfabrik), and group VE (Enamic, Vita Zahnfabrik). In incisal reduction of $1.5 \mathrm{~mm}$ was made with a butt joint margin and $0.6 \mathrm{~mm}$ finish line was prepared on the labial surface of each specimen and on the middle of the proximal surfaces. Each group received different type of veneers according to the sample grouping. 
Aone-step double-consistency silicon impression material was taken for each specimen. Stone dies were poured and individually scanned using digital scanner, where a CAD model was generated for each specimen individually. After milling, veneers where cut off the block, sintered, finished and polished according to manufacturer instructions. Specimens and teeth were surface treated to receive its corresponding veneer and then cemented using light cured resin cement.

Using spectrophotometer (Nippon Densmoku industries), $\Delta \mathrm{E}$ was recorded for each specimen before and after 15 days of immersion in coffee solution. The specimens and the spectrophotometer were always in the same position during all the measurements. Using a spectrophotometer, 3 color parameters were recorded for each ceramic veneer before and after immersion in a coffee solution according to the following equation: $\Delta \mathrm{E}^{*}=[(\Delta \mathrm{L} *) 2+(\Delta \mathrm{a} *) 2+(\Delta \mathrm{b} *) 2] 1 / 2$; where $\Delta \mathrm{L}^{*}$ is the variation of $\mathrm{L}^{*}, \Delta \mathrm{a}^{*}$ is the variation of $\mathrm{a}^{*}$, and $\Delta b^{*}$ is the variation of $b^{*} . \Delta E^{*}$ were obtained in this study for the 3 groups tested. Considering the value of $\Delta \mathrm{E}^{*}>3.3$ as clinically significant staining. Each specimen was then mounted individually in universal testing machine using a cross head speed



Fig. (2) Mean values of $\Delta E$ of the studied groups. of $0.5 \mathrm{~mm} / \mathrm{min}$ at 135 degrees to the long axis of the tooth on palatal surface to simulate the average angle of contact between maxillary and mandibular incisors in class 1 malocclusion, using a specially designed load application jig simulating mandibular central incisors. One-way ANOVA and chi-square tests were used for statistical analysis.

\section{RESULTS}

\section{1- Color stability}

VS group scored the lowest $\Delta \mathrm{E}$ mean value (3.52) followed by VE group (4.14) and the highest value scored by Em group (4.49). One-Way ANOVA test revealed no significant differences between the tested groups $(\mathrm{P}=0.636)$. For clinical acceptability of color changes, Chi-square test was applied to $\Delta \mathrm{E}$ at 3.3 threshold and the results revealed no significant differences between the tested groups $(\mathrm{p}=0.387)$.

\section{2- Fracture resistance}

VS group scored the highest mean value of fracture resistance (471.04) followed Em group (442.13) and lowest value scored by VE groups (394.72). One-Way ANOVA test was applied to studied groups and there were also no significant differences $(\mathrm{P}=0.534)$.



Fig. (3) Mean (Newton) values of fracture strength. 


\section{DISCUSSION}

In current study, certain procedural steps were adopted to ensure proper standardization and that the purpose of the study was fulfilled.

Stone dies were used for scanning without imaging powder, instead of the natural teeth was adopted to prevent powdering the tooth which may interfere with the adhesion of the veneer as recommended. ${ }^{8}$

Light cure resin cement was utilized because of its recommendation for esthetic metal-free restorations (Simon and de Rijk 2006). An advantage of these cements is the increased working time compared to the other cure types ${ }^{9}$ in addition to their color stability. ${ }^{10}$

CIELAB (Commission Internationale de I'Eclairage) color coordinates system is a very useful mode, providing information about location of object color in a uniform 3 dimensional color space as documented by Gupta et al. (2005). ${ }^{11} \mathrm{~A}$ customized plastic box having the same diameter as the measuring tip of the spectrophotometer was fabricated, in order to constrict the readings on the tooth only without reading of the surrounding colours.

Although there were no significant differences between the groups, Suprinity exhibited the best colour stability values $(\Delta \mathrm{E}=3.52)$ followed by Enamic $(\Delta \mathrm{E}=4.14)$ and then e-max $(\Delta \mathrm{E}=4.49)$. This insignificant difference between Enamic, and Suprinity compared with the control group (e-max), may be attributed to the fact that Enamic contains $86 \%$ ceramic filler which may render the material to act like ceramic material than a resin material ${ }^{12}$, as well as the Suprinity which contains only $10 \%$ zirconia fillers. ${ }^{7}$

The high $\Delta \mathrm{E}$ of all the groups could be due to surface roughness of the veneers, as they were only mechanically polished without addition of glazing material. This is in accordance to a recently published research ${ }^{13}$ which revealed that the absorption of extrinsic stain from coffee is affected by surface roughness, integrity, and polishing regime. However, it is hard to relate the results of this study to the clinical conditions as the colour stability of restorative materials cannot be related to a single beverage, but it is the consequence of complex reaction of different chemicals for different food, drinks and mouth rinses. ${ }^{14}$ Further investigations are needed to assess the effect of polishing and glazing of the discoloured composite, and ceramic laminate veneers on the improvement of the discoloration.

Regarding the fracture resistance test, mounting of the specimens was guided using 45 degrees angled cuts on proximal surfaces of the resin blocks which give an interincisal angle of 135 degrees which simulate the angle in class I malocclusion. ${ }^{15}$

Fracture resistance results showed, highest values for Suprinity (471 N) followed by e-max (442 N) and the lowest was for Enamic (394 N) but with no significant differences between them. Several factors may influence the fracture resistance-ceramic restoration such as microstructure and fatigue of the ceramic material, fabrication technique, preparation design and luting method. The slight surpass of Suprinity in the fracture resistance, could be attributed to its composition of zirconia fillers which increase its fracture strength. On the other hand, the approximate values of fracture resistance exhibited by Enamic, compared with the control (e-max), could be due to the existence of polymer in its composition which reduces the brittle fracture and lead to increase fracture resistance. ${ }^{16}$ The large standard deviations obtained in this study could be attributed to various factors, including natural variations in tooth properties and anatomy as well as defects introduced during preparation and the CAM processing or statistical variations regarding sample size.

The null hypothesis was accepted as there was no statistical difference among tested groups. 


\section{CONCLUSION}

Within the limitation of this study it was concluded that,

1- There was no perceivable color difference between Suprinity, Enamic and e-max.

2- There was no significant difference in the fracture resistance between Suprinity, Enamic and e-max.

3- The two tested hybrid ceramics could present an acceptable treatment option for fabricating ceramic veneers compared to e-max.

\section{REFERENCES}

1- Zaninovich, Michael. The effect of clinical polishing protocols on ceramic surface texture and wear rate of opposing enamel: a laboratory study.2014, unpublished $\mathrm{PhD}$ thesis, The University of Adelaide.

2- Giordano RA 2nd, Pelletier L, Campbell S, Pober R. Flexural strength of an infused ceramic, glass ceramic, and feldspathic porcelain. J Prosthet Dent. 1995 May;73(5):411-8.

3- McLaren EA, LeSage B. Feldspathic veneers: what are their indications? Compend Contin Educ Dent. 2011 Apr;32(3):44-9.

4- Silva TM, Salvia AC, Carvalho RF, Pagani C, Rocha DM, Silva EG. Polishing for glass ceramics: which protocol? J Prosthodont Res. 2014 Jul;58(3):160-70.

5- Zarone F, Russo S, Sorrentino R. From porcelain-fusedto-metal to zirconia: clinical and experimental considerations. Dent Mater. 2011 Jan;27(1):83-96.

6- Spitznagel FA, Horvath SD, Guess PC, Blatz MB. Resin bond to indirect composite and new ceramic/polymer materials: a review of the literature. J Esthet Restor Dent. 2014 Nov-Dec;26(6):382-93.

7- Sannino G, Germano F, Arcuri L, Bigelli E, Arcuri C, Barlattani A. CEREC CAD/CAM Chairside System. Oral Im-

plantol (Rome). 2015 Apr 13;7(3):57-70.

8- Alghazzawi TF, Al-Samadani KH, Lemons J, Liu PR, Essig ME, Bartolucci AA, Janowski GM. Effect of imaging powder and CAD/CAM stone types on the marginal gap of zirconia crowns. J Am Dent Assoc. 2015 Feb; 146(2):111-20.

9- Simon JF, Darnell LA. Considerations for proper selection of dental cements. Compend Contin Educ Dent. 2012 Jan;33(1):28-30.

10- Peumans M, Van Meerbeek B, Lambrechts P, Vanherle G. Porcelain veneers: a review of the literature. J Dent. 2000 Mar;28(3):163-77.

11- Gupta, R., Parkash, H., Shah, N., \& Jain, V. (2005). A spectrophotometric evaluation of color changes of various tooth colored veneering materials after exposure to commonly consumed beverages. The Journal of Indian Prosthodontic Society. June 2005; 5(2), 72-78

12- Elsaka SE. Bond strength of novel CAD/CAM restorative materials to self-adhesive resin cement: the effect of surface treatments. J Adhes Dent. 2014 Dec;16(6):531-40.

13- Malekipour MR, Sharafi A, Kazemi S, Khazaei S, Shirani F. Comparison of color stability of a composite resin in different color media. Dent Res J. 2012 Jul;9(4):441-6.

14- Khaledi AAR, Safari A, Adibi A Adibi S. The Effect of Chlorhexidine Mouth Rinse on the Colour Stability of Porcelain with Three Different Surface Treatments: An in Vitro Study. Journal of Dental Biomaterials.2014; ;1(1): 3-8.

15- Kubein D, Jäger A, Paschereit F. [Deviations of the individual interincisal angle and ideal axis position of upper and lower front teeth (comparative study between a dysgnathic group and a normal group)]. Dtsch Zahnarztl Z . 1983 Aug;38(8):752-8.

16- Johnson AC, Versluis A, Tantbirojn D, Ahuja S. Fracture strength of CAD/CAM composite and compositeceramic occlusal veneers. J Prosthodont Res. 2014 Apr; 58(2):107-14. 